ISSN 1855-3966 (printed edn.), ISSN 1855-3974 (electronic edn.)

ARS MATHEMATICA CONTEMPORANEA 18 (2020) 289-307

https://doi.org/10.26493/1855-3974.1748.ebd

(Also available at http://amc-journal.eu)

\title{
Complete regular dessins and skew-morphisms of cyclic groups*
}

\author{
Yan-Quan Feng ${ }^{\dagger}$ \\ Department of Mathematics, Beijing Jiaotong University, \\ Beijing 100044, People's Republic of China
}

$\mathrm{Kan} \mathrm{Hu} \ddagger$

School of Mathematics, Physics and Information Science, Zhejiang Ocean University, Zhoushan, Zhejiang 316022, People's Republic of China

\section{Roman Nedela ${ }^{\S}$}

University of West Bohemia, NTIS FAV, Pilsen, Czech Republic and Mathematical Institute, Slovak Academy of Sciences, Banská Bystrica, Slovakia

\author{
Martin Škoviera $₫$ \\ Department of Computer Science, Comenius University, 84248 Bratislava, Slovakia
}

Na-Er Wang $\|$

Key Laboratory of Oceanographic Big Data Mining \& Application of Zhejiang Province, Zhoushan, Zhejiang 316022, People's Republic of China

Received 8 July 2018, accepted 18 January 2020, published online 21 October 2020

\begin{abstract}
A dessin is a 2-cell embedding of a connected 2-coloured bipartite graph into an orientable closed surface. A dessin is regular if its group of orientation- and colour-preserving automorphisms acts regularly on the edges. In this paper we study regular dessins whose underlying graph is a complete bipartite graph $K_{m, n}$, called $(m, n)$-complete regular dessins. The purpose is to establish a rather surprising correspondence between $(m, n)$ complete regular dessins and pairs of skew-morphisms of cyclic groups. A skew-morphism

\footnotetext{
* The authors would like to express their gratitude to the anonymous referees for their helpful comments and suggestions which have improved the content and presentation of the paper.

${ }^{\dagger}$ National Natural Science Foundation of China (No. 11571035, 11731002).

$\ddagger$ (Corresponding author.) Zhejiang Provincial Natural Science Foundation of China (No. LY16A010010).

$\S$ APVV-15-0220; VEGA 2/0078/20; Project LO1506 of the Czech Ministry of Education, Youth and Sports.

ฯAPVV-15-0220; VEGA 1/0813/18.

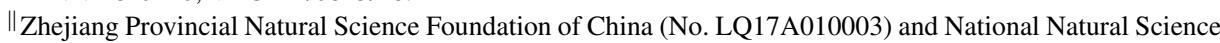
Foundation of China (No. 11801507).
\end{abstract}

(c)(7) This work is licensed under https://creativecommons.org/licenses/by/4.0/ 
of a finite group $A$ is a bijection $\varphi: A \rightarrow A$ that satisfies the identity $\varphi(x y)=\varphi(x) \varphi^{\pi(x)}(y)$ for some function $\pi: A \rightarrow \mathbb{Z}$ and fixes the neutral element of $A$. We show that every $(m, n)$-complete regular dessin $\mathcal{D}$ determines a pair of reciprocal skew-morphisms of the cyclic groups $\mathbb{Z}_{n}$ and $\mathbb{Z}_{m}$. Conversely, $\mathcal{D}$ can be reconstructed from such a reciprocal pair. As a consequence, we prove that complete regular dessins, exact bicyclic groups with a distinguished pair of generators, and pairs of reciprocal skew-morphisms of cyclic groups are all in a one-to-one correspondence. Finally, we apply the main result to determining all pairs of integers $m$ and $n$ for which there exists, up to interchange of colours, exactly one isomorphism class of $(m, n)$-complete regular dessins. We show that the latter occurs precisely when every group expressible as a product of cyclic groups of order $m$ and $n$ is abelian, which eventually comes down to the condition $\operatorname{gcd}(m, \phi(n))=\operatorname{gcd}(\phi(m), n)=$ 1 , where $\phi$ is Euler's totient function.

Keywords: Regular dessin, bicyclic group, skew-morphism, graph embedding.

Math. Subj. Class. (2020): 05E18, 20B25, 57M15

\section{Introduction}

A dessin is a cellular embedding $i: \Gamma \hookrightarrow \mathcal{C}$ of a connected bipartite graph $\Gamma$, endowed with a fixed proper 2-colouring of its vertices, into an orientable closed surface $\mathcal{C}$ such that each component of $\mathcal{C} \backslash i(\Gamma)$ is homeomorphic to the open disc. An automorphism of a dessin is a colour-preserving automorphism of the underlying graph that extends to an orientationpreserving self-homeomorphism of the supporting surface. The action of the automorphism group of a dessin on the edges is well known to be semi-regular; if this action is transitive, and hence regular, the dessin itself is called regular.

Dessins - more precisely dessins d'enfants - were introduced by Grothendieck in [42] as a combinatorial counterpart of algebraic curves. Grothendieck was inspired by a theorem of Belyi [3] which states that a compact Riemann surface $\mathcal{C}$, regarded as a projective algebraic curve, can be defined by an algebraic equation $P(x, y)=0$ with coefficients from the algebraic number field $\overline{\mathbb{Q}}$ if and only if there exists a non-constant meromorphic function $\beta: \mathcal{C} \rightarrow \mathbb{P}^{1}(\mathbb{C})$, branched over at most three points, which can be chosen to be 0,1 , and $\infty$. It follows that each such curve carries a dessin in which the black and the white vertices are the preimages of 0 and 1 , respectively, and the edges are the preimages of the unit interval $I=[0,1]$. The absolute Galois group $\mathbb{G}=\operatorname{Gal}(\overline{\mathbb{Q}} / \mathbb{Q})$ has a natural action on the curves and thus also on the dessins. As was shown by Grothendieck [42], the action of $\mathbb{G}$ on dessins is faithful. More recently, González-Diez and Jaikin-Zapirain [13] have proved that this action remains faithful even when restricted to regular dessins. It follows that one can study the absolute Galois group through its action on such simple and symmetrical combinatorial objects as regular dessins.

In this paper we study regular dessins whose underlying graph is a complete bipartite graph $K_{m, n}$, which we call complete regular dessins, or more specifically $(m, n)$-complete regular dessins. The associated algebraic curves may be viewed as a generalisation of the Fermat curves, defined by the equation $x^{n}+y^{n}=1$ (see Lang [38]). These curves have recently attracted considerable attention, see for example [7, 24, 25, 27, 28]. Classification

E-mail addresses: yqfeng@bjtu.edu.cn (Yan-Quan Feng), hukan@zjou.edu.cn (Kan Hu), nedela@savbb.sk (Roman Nedela), skoviera@dcs.fmph.uniba.sk (Martin Škoviera), wangnaer@zjou.edu.cn (Na-Er Wang) 
of complete regular dessins is therefore a very natural problem, interesting from algebraic, combinatorial, and geometric points of view.

Jones, Nedela, and Škoviera [23] were first to observe that there is a correspondence between complete regular dessins and exact bicyclic groups. Recall that a finite group $G$ is bicyclic if it can be expressed as a product $G=A B$ of two cyclic subgroups $A$ and $B$; if the two subgroups are disjoint, that is, if $A \cap B=\{1\}$, the bicyclic group is called exact. Exact bicyclic groups are, in turn, closely related to skew-morphisms of the cyclic groups.

A skew-morphism of a finite group $A$ is a bijection $\varphi: A \rightarrow A$ fixing the identity element of $A$ and obeying the morphism-type rule $\varphi(x y)=\varphi(x) \varphi^{\pi(x)}(y)$ for some integer function $\pi: A \rightarrow \mathbb{Z}$. In the case where $\pi$ is the constant function $\pi(x)=1$, a skewmorphism is just an automorphism. Thus, skew-morphisms may be viewed as a generalisation of group automorphisms. The concept of skew-morphism was introduced by Jajcay and Širán as an algebraic tool to the investigation of (orientably) regular Cayley maps. In the seminal paper [20] they proved that a Cayley map $\operatorname{CM}(A, X, P)$ of a finite group $A$ is regular if and only if there is a skew-morphism of $A$ such that the restriction of $\varphi$ to $X$ is equal to $P$ [20, Theorem 1]. Thus the classification problem of regular Cayley maps of a finite group $A$ is reduced to a problem of determining certain skew-morphisms of $A$. The interested reader is referred to [5, 6, 29, 30, 31, 34, 35, 36, 46, 47] for progress in this direction.

The main purpose of this paper is to establish another surprising connection between skew-morphisms and complete regular dessins. As we have already mentioned above, every $(m, n)$-complete regular dessin can be represented as an exact bicyclic group factorisation $G=\langle a\rangle\langle b\rangle$ with two distinguished generators $a$ and $b$ of orders $m$ and $n$, respectively (see [23]). The factorisation gives rise to a pair of closely related skew-morphisms of cyclic groups $\varphi: \mathbb{Z}_{n} \rightarrow \mathbb{Z}_{n}$ and $\varphi^{*}: \mathbb{Z}_{m} \rightarrow \mathbb{Z}_{m}$ which satisfy two simple technical conditions (see Definition 3.2); such a pair of skew-morphisms will be called reciprocal. We prove that isomorphic complete regular dessins give rise to the same pair of reciprocal skewmorphisms, which is a rather remarkable fact, because every complete regular dessin thus receives a natural algebraic invariant.

Even more surprising is the fact that given a pair of reciprocal skew-morphisms $\varphi: \mathbb{Z}_{n} \rightarrow \mathbb{Z}_{n}$ and $\varphi^{*}: \mathbb{Z}_{m} \rightarrow \mathbb{Z}_{m}$, one can reconstruct the original complete regular dessin up to isomorphism. In other words, a pair of reciprocal skew-morphisms of the cyclic groups constitutes a complete set of invariants for a regular dessin whose underlying graph is the complete bipartite graph. One can therefore study and classify complete regular dessins by means of determining pairs of reciprocal skew-morphisms of cyclic groups. Note that the classification of skew-morphisms of the cyclic groups is a prominent open problem, see [1, 2, 5, 6, 32, 33] for partial results.

The relationship between complete regular dessins and exact bicyclic groups has an important implication for the classical classification problem of bicyclic groups in group theory (see $[8,16,18,21])$. More precisely, suppose that we are given an exact product $G=A B$ of two cyclic groups $A$ and $B$ with distinguished generators $a \in A$ and $b \in B$. The corresponding pair of reciprocal skew-morphisms $\left(\varphi, \varphi^{*}\right)$ and associated pair of power functions $\left(\pi, \pi^{*}\right)$ can be alternatively derived from the equations

$$
b a^{x}=a^{\varphi(x)} b^{\pi(x)} \quad \text { and } \quad a b^{y}=b^{\varphi^{*}(y)} a^{\pi^{*}(y)},
$$

and thus encodes the commuting rules within $G$. By our main result, determining all exact bicyclic groups with a distinguished generator pair is equivalent to determining all pairs 
of reciprocal skew-morphisms. Thus to describe all exact bicyclic groups it is sufficient to characterise all pairs of reciprocal skew-morphisms of the cyclic groups.

Our paper is organised as follows. In Section 2 we describe the basic correspondence between complete regular dessins and exact bicyclic triples $(G ; a, b)$, where $G$ is a group which factorises as $G=\langle a\rangle\langle b\rangle$ with $\langle a\rangle \cap\langle b\rangle=\{1\}$. Given a complete regular dessin $\mathcal{D}$, its automorphism group $G=\operatorname{Aut}(\mathcal{D})$ can be factorised as a product of two disjoint cyclic subgroups $\langle a\rangle$ and $\langle b\rangle$ where $\langle a\rangle$ is the stabiliser of one black vertex and $\langle b\rangle$ is the stabiliser of one white vertex. The triple $(G ; a, b)$ is then an exact bicyclic triple. Conversely, each exact bicyclic triple $(G ; a, b)$ determines a complete regular dessin where the elements of $G$ are the edges, the cosets of $\langle a\rangle$ are black vertices, the cosets of $\langle b\rangle$ are white vertices, and the local rotations at black and white vertices, respectively, correspond to the multiplication by $a$ and $b$.

In Section 3 we introduce the concept of a reciprocal skew-morphism and prove the main result, Theorem 3.5, which establishes the aforementioned correspondence between complete regular dessins and pairs of reciprocal skew-morphisms of cyclic groups.

An important part of the classification of complete regular dessins is identifying all pairs of integers $m$ and $n$ for which there exists a unique complete regular dessin up to isomorphism and interchange of colours. This problem will be discussed in Section 4. In view of the correspondence between complete regular dessins and pairs of reciprocal skewmorphisms of cyclic groups, we ask for which integers $m$ and $n$ the only reciprocal pair of skew-morphisms is the trivial pair formed by the two identity automorphisms. In other words, we wish to determine all pairs of positive integers $m$ and $n$ that give rise to only one exact product of cyclic groups $\mathbb{Z}_{m}$ and $\mathbb{Z}_{m}$, which necessarily must be the direct product $\mathbb{Z}_{m} \times \mathbb{Z}_{n}$. The answer is given in Theorem 4.4 which states that all this occurs precisely when $\operatorname{gcd}(m, \phi(n))=\operatorname{gcd}(\phi(m), n)=1$, where $\phi$ is the Euler's totient function. This theorem presents six equivalent conditions one of which corresponds to a recent result of Fan and Li [12] about the existence of a unique edge-transitive orientable embedding of a complete bipartite graph. While the proof in [12] is based on the structure of exact bicyclic groups, our proof employs the correspondence theorems established in Section 3.

Theorem 4.4 is a direct generalisation of a result of Jones, Nedela, and Škoviera [23] where it is assumed that the complete dessin in question admits an external symmetry swapping the two partition sets. Theorem 4.4 also strengthens the main result of [12] by extending it to all products of cyclic groups rather than just to those where the intersection of factors is trivial. In particular, we prove that every group that factorises as a product of two cyclic subgroups of orders $m$ and $n$ is abelian if and only if $\operatorname{gcd}(m, \phi(n))=$ $\operatorname{gcd}(\phi(m), n)=1$, where $\phi$ is Euler's totient function. This generalises an old result due to Burnside which states that every group of order $n$ is cyclic if and only if $\operatorname{gcd}(n, \phi(n))=1$, see $[41, \S 10.1]$.

Finally, in Section 5 we deal with the symmetric case, that is, with the case where the reciprocal skew-morphism pairs have the form $(\varphi, \varphi)$. In this situation, the corresponding complete regular dessins admit an additional external symmetry transposing the two partition sets, and thus are essentially the same thing as orientably regular embeddings of the complete bipartite graphs $K_{n, n}$ recently classified in a series of papers [9, 10, 11, 23, 25, 26, 40]. 


\section{Complete regular dessins}

It is well known that every dessin, as defined in the previous section, can be regarded as a two-generator transitive permutation group acting on a non-empty finite set [24]. Given a dessin $\mathcal{D}$ on an oriented surface $\mathcal{C}$, we can define two permutations $\rho$ and $\lambda$ on the edge set of $\mathcal{D}$ as follows: For every black vertex $v$ and every white vertex $w$ let $\rho_{v}$ and $\lambda_{w}$ be the cyclic permutations of edges incident with $v$ or $w$, respectively, induced by the orientation of $\mathcal{C}$. Set $\rho=\prod_{v} \rho_{v}$ and $\lambda=\prod_{w} \lambda_{w}$, where $v$ and $w$ run through the set of all black and white vertices, respectively. Since the underlying graph of $\mathcal{D}$ is connected, the group $G=\langle\rho, \lambda\rangle$ is transitive. Conversely, given a transitive permutation group $G=\langle\rho, \lambda\rangle$ acting on a finite set $\Omega$, we can reconstruct a dessin $\mathcal{D}$ as follows: Take $\Omega$ to be the edge set of $\mathcal{D}$, the orbits of $\rho$ to be the black vertices, and the orbits of $\lambda$ to be white vertices, with incidence being defined by containment. The vertices and edges of $\mathcal{D}$ clearly form a bipartite graph $\Gamma$, the underlying graph of $\mathcal{D}$. The underlying graph is connected, because the action of $G$ on $\Omega$ is transitive. The cycles of $\rho$ and $\lambda$ determine the local rotations around black and white vertices, respectively, thereby giving rise to a 2 -cell embedding of $\Gamma$ into an oriented surface. Summing up, we can identify a dessin with a triple $(\Omega ; \rho, \lambda)$ where $\Omega$ is a nonempty finite set, and $\rho$ and $\lambda$ are permutations of $\Omega$ such that the group $\langle\rho, \lambda\rangle$ is transitive on $\Omega$; this group is called the monodromy group of $\mathcal{D}$ and is denoted by $\operatorname{Mon}(\mathcal{D})$.

Two dessins $\mathcal{D}_{1}=\left(\Omega_{1} ; \rho_{1}, \lambda_{1}\right)$ and $\mathcal{D}_{2}=\left(\Omega_{2} ; \rho_{2}, \lambda_{2}\right)$ are isomorphic provided that there is a bijection $\alpha: \Omega_{1} \rightarrow \Omega_{2}$ such that $\alpha \rho_{1}=\rho_{2} \alpha$ and $\alpha \lambda_{1}=\lambda_{2} \alpha$. An isomorphism of a dessin $\mathcal{D}$ to itself is an automorphism of $\mathcal{D}$. It follows that the automorphism group $\operatorname{Aut}(\mathcal{D})$ of $\mathcal{D}$ is the centraliser of $\operatorname{Mon}(\mathcal{D})=\langle\rho, \lambda\rangle$ in the symmetric group $\operatorname{Sym}(\Omega)$. As $\operatorname{Mon}(\mathcal{D})$ is transitive, $\operatorname{Aut}(\mathcal{D})$ is semi-regular on $\Omega$. If $\operatorname{Aut}(\mathcal{D})$ is transitive, and hence regular on $\Omega$, the dessin $\mathcal{D}$ itself is called regular.

Since every regular action of a group on a set is equivalent to its action on itself by multiplication, every regular dessin can be identified with a triple $\mathcal{D}=(G ; a, b)$ where $G$ is a finite group generated by two elements $a$ and $b$. Given such a triple $\mathcal{D}=(G ; a, b)$, we can define the edges of $\mathcal{D}$ to be the elements of $G$, the black vertices to be the left cosets of the cyclic subgroup $\langle a\rangle$, and the white vertices to be the left cosets of the cyclic subgroup $\langle b\rangle$. An edge $g \in G$ joins the vertices $s\langle a\rangle$ and $t\langle b\rangle$ if and only if $g \in s\langle a\rangle \cap t\langle b\rangle$. In particular, the underlying graph is simple if and only if $\langle a\rangle \cap\langle b\rangle=\{1\}$. The local rotation of edges around a black vertex $s\langle a\rangle$ corresponds to the right translation by the generator $a$, that is, $s a^{i} \mapsto s a^{i+1}$ for any integer $i$. Similarly, the local rotation of edges around a white vertex $t\langle b\rangle$ corresponds to the right translation by the generator $b$, that is, $t b^{i} \mapsto t b^{i+1}$ for any integer $i$. It follows that $\operatorname{Mon}(\mathcal{D})$ can be identified with the group of all right translations of $G$ by the elements of $G$ while $\operatorname{Aut}(\mathcal{D})$ can be identified with the group of all left translations of $G$ by the elements of $G$. In particular, $\operatorname{Mon}(\mathcal{D}) \cong \operatorname{Aut}(\mathcal{D}) \cong G$ for every regular dessin $\mathcal{D}$.

It is easy to see that two regular dessins $\mathcal{D}_{1}=\left(G_{1} ; a_{1}, b_{1}\right)$ and $\mathcal{D}_{2}=\left(G_{2} ; a_{2}, b_{2}\right)$ are isomorphic if and only if the triples $\left(G_{1} ; a_{1}, b_{1}\right)$ and $\left(G_{2} ; a_{2}, b_{2}\right)$ are equivalent, that is, whenever there is a group isomorphism $G_{1} \rightarrow G_{2}$ such that $a_{1} \mapsto a_{2}$ and $b_{1} \mapsto b_{2}$. Consequently, for a given two-generator group $G$, the isomorphism classes of regular dessins $\mathcal{D}$ with $\operatorname{Aut}(\mathcal{D}) \cong G$ are in a one-to-one correspondence with the orbits of the action of $\operatorname{Aut}(G)$ on the generating pairs $(a, b)$ of $G$.

Following Lando and Zvonkin [37], for a regular dessin $\mathcal{D}=(G ; a, b)$ we define its reciprocal dessin to be the regular dessin $\mathcal{D}^{*}=(G ; b, a)$. Topologically, $\mathcal{D}^{*}$ arises from $\mathcal{D}$ 
simply by interchanging the vertex colours of $\mathcal{D}$. Thus the reciprocal dessin has the same underlying graph, the same supporting surface, and the same automorphism group as the original one. Clearly, $\mathcal{D}^{*}$ is isomorphic to $\mathcal{D}$ if and only if $G$ has an automorphism swapping the generators $a$ and $b$. If this occurs, the regular dessin $\mathcal{D}$ will be called symmetric. A symmetric dessin possesses an external symmetry which transposes the vertex-colours and thus is essentially the same thing as an orientably regular bipartite map.

In this paper we apply the general theory to regular dessins whose underlying graph is a complete bipartite graph. A regular dessin $\mathcal{D}$ will be called an $(m, n)$-complete regular dessin, or simply a complete regular dessin, if its underlying graph is the complete bipartite graph $K_{m, n}$ whose $m$-valent vertices are coloured black and $n$-valent vertices are coloured white. If $\mathcal{D}$ is an $(m, n)$-complete regular dessin, then the reciprocal dessin $\mathcal{D}^{*}$ is an $(n, m)$ complete regular dessin. Thus all complete regular dessins appear in reciprocal pairs. Note that $m=n$ does not necessarily imply that the dessin is symmetric.

Complete regular dessins can be easily described in group theoretical terms: their automorphism group is just an exact bicyclic group. This fact was first observed by Jones et al. in [23]. A bicyclic group $G=\langle a\rangle\langle b\rangle$ with $|a|=m$ and $|b|=n$ will be called an $(m, n)$ bicyclic group and $(G ; a, b)$ an $(m, n)$-bicyclic triple. Note that an exact $(m, n)$-bicyclic group has precisely $m n$ elements.

The following statement was proved by Jones, Nedela, and Škoviera in [23, Section 2] under the condition that $m=n$. However, the same arguments can be used to prove it for any $m$ and $n$, so we state it without proof.

Theorem 2.1. A regular dessin $\mathcal{D}=(G ; a, b)$ is complete if and only if $G=\langle a\rangle\langle b\rangle$ is an exact bicyclic group. Furthermore, the isomorphism classes of $(m, n)$-complete regular dessins are in a one-to-one correspondence with the equivalence classes of exact $(m, n)$ bicyclic triples.

Example 2.2. For each pair of positive integers $m$ and $n$ there is an exact bicyclic triple $(G ; a, b)$ where

$$
G=\left\langle a, b \mid a^{m}=b^{n}=[a, b]=1\right\rangle=\langle a\rangle \times\langle b\rangle \cong \mathbb{Z}_{m} \times \mathbb{Z}_{n}
$$

with $[a, b]$ denoting the commutator $a^{-1} b^{-1} a b$. It is easy to see that this triple is uniquely determined by the group $\mathbb{Z}_{m} \times \mathbb{Z}_{n}$ up to order of generators and equivalence, so up to reciprocality this group gives rise to a unique complete regular dessin with underlying graph $K_{m, n}$. We call this dessin the standard $(m, n)$-complete dessin. If $m=n$, the group $G$ has an automorphism transposing $a$ and $b$, which implies that in this case the dessin is symmetric. The corresponding embedding is the standard embedding of $K_{n, n}$ described in [23, Example 1]. The associated algebraic curves coincide with the Fermat curves.

\section{Reciprocal skew-morphisms}

In this section we establish a correspondence between exact bicyclic triples and certain pairs of skew-morphisms of cyclic groups.

Recall that a skew-morphism $\varphi$ of a finite group $A$ is a bijection $A \rightarrow A$ fixing the identity of $A$ for which there exists an associated power function $\pi: A \rightarrow \mathbb{Z}$ such that

$$
\varphi(x y)=\varphi(x) \varphi^{\pi(x)}(y)
$$


for all $x, y \in A$. It may be useful to realise that $\pi$ is not uniquely determined by $\varphi$. However, if $\varphi$ has order $d$, then $\pi$ can be regarded as a function $A \rightarrow \mathbb{Z}_{d}$, which is unique. In the special case where $\pi(x)=1$ for all $x \in A, \varphi$ is a group automorphism. In general, the set $\{x \in A ; \pi(x)=1\}$ is a subgroup of $A$, called the kernel of $\varphi$ and denoted by $\operatorname{ker} \varphi$.

Skew-morphisms have a number of important properties, sometimes very different from those of group automorphisms. In our treatment we restrict ourselves to a few basic properties of skew-morphisms needed in this paper. For a more detailed account we refer the reader to [5, 20, 32, 45, 48].

The next three properties of skew-morphisms are well known and were proved in [20, Lemma 2], [19, Lemma 2.1], and [45, Lemma 2.6], respectively.

Lemma 3.1. Let $\varphi$ be a skew-morphism of a finite group A with associated power function $\pi$. Let $d$ be the order of $\varphi$. Then:

(i) for any two elements $x, y \in A$ and an arbitrary positive integer $k$ one has

$$
\varphi^{k}(x y)=\varphi^{k}(x) \varphi^{\sigma(x, k)}(y) \quad \text { where } \quad \sigma(x, k)=\sum_{i=1}^{k} \pi\left(\varphi^{i-1}(x)\right) ;
$$

(ii) for every element $x \in A$ one has $\mathcal{O}_{x^{-1}}=\mathcal{O}_{x}^{-1}$, where $\mathcal{O}_{x}$ denotes the orbit of $\varphi$ containing $x$;

(iii) for every $x \in A$ one has $\sigma(x, d) \equiv 0(\bmod d)$.

Let $G$ be a finite group which is expressible as a product $A C$ of two subgroups $A$ and $C$ where $C$ is cyclic and $A \cap C=\{1\}$; in this situation we say that $C$ is a cyclic complement of $A$. Choose a generator $c$ of $C$. Since $G=A C=C A$, for every element $x \in A$ we can write the product $c x$ in the form $y c^{k}$, so

$$
c x=y c^{k}
$$

for some $y \in A$ and $k \in \mathbb{Z}_{|c|}$. Note that both $y \in A$ and $k \in \mathbb{Z}_{|c|}$ are uniquely determined by $x$. Thus we can define functions $\varphi_{c}: A \rightarrow A$ and $\pi_{c}: A \rightarrow \mathbb{Z}_{|c|}$ by setting

$$
\varphi_{c}(x)=y \quad \text { and } \quad \pi_{c}(x)=k .
$$

It is not difficult to verify that $\varphi_{c}$ is a skew-morphism of $A$ and $\pi_{c}$ is an associated power function (see [4, p. 262] or [5, p. 73]). We call $\varphi_{c}$ the skew-morphism induced by $c$. The order $\left|\varphi_{c}\right|$ of this skew-morphism equals the index $\left|\langle c\rangle:\langle c\rangle_{G}\right|$ where $\langle c\rangle_{G}=\cap_{g \in G}\langle c\rangle^{g}$; see [5, Lemma 4.1]. It follows that the power function $\pi_{c}$ can be further reduced to a function $A \rightarrow \mathbb{Z}_{\left|\varphi_{c}\right|}$, still denoted by $\pi_{c}$.

We now focus on the particular case $G=A B$ where both $A$ and $B$ are cyclic and $A \cap B=\{1\}$, which means that $G$ is an exact bicyclic group. The subgroups $A$ and $B$ can now be taken as cyclic complements of each other. Therefore a generator $a$ of $A$ induces a skew-morphism of $B$ and a generator $b$ of $B$ induces a skew-morphism of $A$. In other words, every exact bicyclic triple $(G ; a, b)$ gives rise to a pair of skew-morphisms, one for each of the two cyclic subgroups.

Next we show that this pair of skew-morphisms can be characterised by two simple properties. For this purpose, we need the following definition. We switch to the additive notation. 
Definition 3.2. A pair $\left(\varphi, \varphi^{*}\right)$ of skew-morphisms $\varphi: \mathbb{Z}_{n} \rightarrow \mathbb{Z}_{n}$ and $\varphi^{*}: \mathbb{Z}_{m} \rightarrow \mathbb{Z}_{m}$ with power functions $\pi$ and $\pi^{*}$, respectively, will be called $(m, n)$-reciprocal if the following two conditions are satisfied:

(i) $|\varphi|$ divides $m$ and $\left|\varphi^{*}\right|$ divides $n$,

(ii) $\pi(x)=-\varphi^{*-x}(-1)$ and $\pi^{*}(y)=-\varphi^{-y}(-1)$ are power functions for $\varphi$ and $\varphi^{*}$, respectively.

If $m=n$ and $\left(\varphi, \varphi^{*}\right)$ is an $(n, n)$-reciprocal pair of skew-morphisms, it may, but need not, happen that $\varphi=\varphi^{*}$. If it does, then the pair $(\varphi, \varphi)$, as well as the skew-morphism $\varphi$ itself, will be called symmetric. Note that a skew-morphism $\varphi$ of $\mathbb{Z}_{n}$ is symmetric if and only if $|\varphi|$ divides $n$ and $\pi(x)=-\varphi^{-x}(-1)$ is a power function of $\varphi$.

Proposition 3.3. If $(G ; a, b)$ is an exact $(m, n)$-bicyclic triple with $\langle a\rangle \cong \mathbb{Z}_{m}$ and $\langle b\rangle \cong$ $\mathbb{Z}_{n}$, then the pair of induced skew-morphisms $\left(\varphi_{a}, \varphi_{b}\right)$ is an $(m, n)$-reciprocal pair of skewmorphisms. If, in addition, $G$ has an automorphism transposing $a$ and b, then $\varphi_{a}=\varphi_{b}$ and the pair is symmetric.

Proof. Let $\varphi=\varphi_{a}$ and $\varphi^{*}=\varphi_{b}$ be the skew-morphisms of the cyclic groups $\mathbb{Z}_{n}$ and $\mathbb{Z}_{m}$ determined by the identities

$$
a b^{x}=b^{\varphi(x)} a^{\pi(x)} \quad \text { and } \quad b a^{y}=a^{\varphi^{*}(y)} b^{\pi^{*}(y)}
$$

where $\pi=\pi_{a}$ and $\pi^{*}=\pi_{b}$ are the power functions associated with $\varphi$ and $\varphi^{*}$, respectively, and the elements $x \in \mathbb{Z}_{n}$ and $y \in \mathbb{Z}_{m}$ are arbitrary. As mentioned above, the orders of $\varphi$ and $\varphi^{*}$ coincide with the indices $\left|\langle a\rangle: \bigcap_{g \in G}\langle a\rangle^{g}\right|$ and $\left|\langle b\rangle: \bigcap_{g \in G}\langle b\rangle^{g}\right|$ [5, Lemma 4.1]. Hence $|\varphi|$ divides $|\langle a\rangle|=m$ and $\left|\varphi^{*}\right|$ divides $|\langle b\rangle|=n$.

By applying induction to the equations (3.2) we get

$$
a^{k} b^{x}=b^{\varphi^{k}(x)} a^{\sigma(x, k)} \quad \text { and } \quad b^{l} a^{y}=a^{\varphi^{* l}(y)} b^{\sigma^{*}(y, l)},
$$

where

$$
\sigma(x, k)=\sum_{i=1}^{k} \pi\left(\varphi^{i-1}(x)\right) \quad \text { and } \quad \sigma^{*}(y, l)=\sum_{i=1}^{l} \pi^{*}\left(\varphi^{* i-1}(y)\right) .
$$

By inverting these identities we obtain

$$
b^{-x} a^{-k}=a^{-\sigma(x, k)} b^{-\varphi^{k}(x)} \quad \text { and } \quad a^{-y} b^{-l}=b^{-\sigma^{*}(y, l)} a^{-\varphi^{* l}(y)} .
$$

The first equation of (3.3) with $x=-1$ and $k=-y$ yields $b a^{y}=a^{-\sigma(-1,-y)} b^{-\varphi^{-y}(-1)}$, which we compare with the rule $b a^{y}=a^{\varphi^{*}(y)} b^{\pi^{*}(y)}$ and get

$$
a^{\varphi^{*}(y)} b^{\pi^{*}(y)}=a^{-\sigma(-1,-y)} b^{-\varphi^{-y}(-1)} .
$$

Consequently $\pi^{*}(y)=-\varphi^{-y}(-1)$. Similarly, inserting $y=-1$ and $l=-x$ into the second equation of (3.3) we get $a b^{x}=b^{-\sigma^{*}(-1,-x)} a^{-\varphi^{*-x}(-1)}$, and combining this with the rule $a b^{x}=b^{\varphi(x)} a^{\pi(x)}$ we derive $\pi(x)=-\varphi^{*-x}(-1)$. Hence, the pair $\left(\varphi, \varphi^{*}\right)$ is $(m, n)$-reciprocal.

Finally, if $G$ has an automorphism $\theta$ transposing $a$ and $b$, then clearly $m=n$. By applying $\theta$ to the identity $b a^{x}=a^{\varphi^{*}(x)} b^{\pi^{*}(x)}$ we obtain $a b^{x}=\theta\left(b a^{x}\right)=\theta\left(a^{\varphi^{*}(x)} b^{\pi^{*}(x)}\right)=$ $b^{\varphi^{*}(x)} a^{\pi^{*}(x)}$. If we compare the last identity with the rule $a b^{x}=b^{\varphi(x)} a^{\pi(x)}$ we obtain $\varphi^{*}=\varphi$, which means that $\varphi$ is a symmetric skew-morphism of $\mathbb{Z}_{n}$, as required. 
We have just shown that every exact $(m, n)$-bicyclic triple determines an $(m, n)$-reciprocal pair of skew-morphisms. Our next aim is to show that the converse is also true. Let $\left(\varphi, \varphi^{*}\right)$ be an $(m, n)$-reciprocal pair of skew-morphisms of $\mathbb{Z}_{n}$ and $\mathbb{Z}_{m}$ with power functions $\pi$ and $\pi^{*}$, respectively. For the sake of clarity we relabel the elements of $\mathbb{Z}_{n}$ and $\mathbb{Z}_{m}$ by setting

$$
\mathbb{Z}_{n}=\{0,1, \ldots,(n-1)\} \quad \text { and } \quad \mathbb{Z}_{m}=\left\{0^{\prime}, 1^{\prime}, \ldots,(m-1)^{\prime}\right\},
$$

so that $\mathbb{Z}_{n} \cap \mathbb{Z}_{m}=\emptyset$. Let

$$
\rho=(0,1, \ldots,(n-1)) \text { and } \rho^{*}=\left(0^{\prime}, 1^{\prime}, \ldots,(m-1)^{\prime}\right)
$$

denote the cyclic shifts in $\mathbb{Z}_{n}$ and $\mathbb{Z}_{m}$, respectively. We now extend the permutations $\varphi, \rho$, $\varphi^{*}$, and $\rho^{*}$ to the set $\mathbb{Z}_{n} \cup \mathbb{Z}_{m}$ in a natural way, and define a permutation group acting on the set $\mathbb{Z}_{m} \cup \mathbb{Z}_{n}$ by

$$
G=\langle a, b\rangle, \quad \text { where } a=\varphi \rho^{*} \text { and } b=\varphi^{*} \rho .
$$

If we regard $\mathbb{Z}_{m} \cup \mathbb{Z}_{n}$ as the vertex set of the complete bipartite graph $K_{m, n}$ with natural bipartition, it becomes obvious that $G \leq \operatorname{Aut}\left(K_{m, n}\right)$. The following result shows that $G$ is in fact isomorphic to the automorphism group of an $(m, n)$-complete regular dessin.

Proposition 3.4. Given an $(m, n)$-reciprocal pair of skew-morphisms $\left(\varphi, \varphi^{*}\right)$, the triple $(G ; a, b)$, where $a=\varphi \rho^{*}$ and $b=\varphi^{*} \rho$ are permutations acting on the disjoint union $\mathbb{Z}_{m} \cup \mathbb{Z}_{m}$, is an exact $(m, n)$-bicyclic triple. Furthermore, for the skew-morphisms induced by $a$ and $b$ in the triple $(G ; a, b)$ we have $\varphi_{a}=\varphi$ and $\varphi_{b}=\varphi^{*}$.

Proof. Let $\varphi: \mathbb{Z}_{n} \rightarrow \mathbb{Z}_{n}$ and $\varphi^{*}: \mathbb{Z}_{m} \rightarrow \mathbb{Z}_{m}$ be an $(m, n)$-reciprocal pair of skewmorphisms. The definition of reciprocality requires $|\varphi|$ to divide $m$ and $\left|\varphi^{*}\right|$ to divide $n$. Since $\varphi, \rho \in \operatorname{Sym}\left(\mathbb{Z}_{n}\right)$ and $\varphi^{*}, \rho^{*} \in \operatorname{Sym}\left(\mathbb{Z}_{m}\right)$ where $\mathbb{Z}_{m} \cap \mathbb{Z}_{n}=\emptyset$, we see that $\left[\varphi, \rho^{*}\right]=1$ and $\left[\varphi^{*}, \rho\right]=1$. It follows that the elements $a=\varphi \rho^{*}$ and $b=\varphi^{*} \rho$ have orders $|a|=m$ and $|b|=n$. Further, if $x \in\langle a\rangle \cap\langle b\rangle$, then $a^{i}=x=b^{j}$ for some integers $i$ and $j$, so $\left(\varphi \rho^{*}\right)^{i}=\left(\varphi^{*} \rho\right)^{j}$. Thus $\varphi^{i} \rho^{* i}=\rho^{j} \varphi^{* j}$, and hence $\varphi^{i}=\rho^{j}$ and $\rho^{* i}=\varphi^{* j}$. Since $\varphi(0)=0$ and $\rho$ is a full cycle, we have $n \mid j$ and $m \mid i$, and hence $x=1$. Therefore $\langle a\rangle \cap\langle b\rangle=\{1\}$.

Next we show that $\langle a\rangle\langle b\rangle$ is a subgroup of $G$. It is sufficient to verify that $\langle a\rangle\langle b\rangle=$ $\langle b\rangle\langle a\rangle$. For this purpose we need to show that for all $x \in \mathbb{Z}_{n}$ and $y \in \mathbb{Z}_{m}$ there exist numbers $\alpha(x), \beta(x), \alpha^{*}(y)$ and $\beta^{*}(y)$ such that the following commuting rules hold:

$$
a b^{x}=b^{\alpha(x)} a^{\beta(x)} \quad \text { and } \quad b a^{y}=a^{\alpha^{*}(y)} b^{\beta^{*}(y)} .
$$

Substituting $\varphi \rho^{*}$ and $\varphi^{*} \rho$ for $a$ and $b$ we see that the equations in (3.4) are equivalent to the following four equations:

$$
\begin{aligned}
\varphi \rho^{x} & =\rho^{\alpha(x)} \varphi^{\beta(x)}, & \rho^{*} \varphi^{* x} & =\varphi^{* \alpha(x)} \rho^{* \beta(x)} ; \\
\varphi^{*} \rho^{* y} & =\rho^{* \alpha^{*}(y)} \varphi^{* \beta^{*}(y)}, & \rho \varphi^{y} & =\varphi^{\alpha^{*}(y)} \rho^{\beta^{*}(y)} .
\end{aligned}
$$

Since $\varphi$ and $\varphi^{*}$ are skew-morphisms and $\pi$ and $\pi^{*}$ are the associated power functions, for all $i \in \mathbb{Z}_{n}$ and $j \in \mathbb{Z}_{m}$ we have

$$
\begin{aligned}
\varphi \rho^{x}(i) & =\varphi(x+i)=\varphi(x)+\varphi^{\pi(x)}(i)=\rho^{\varphi(x)} \varphi^{\pi(x)}(i) \\
\varphi^{*} \rho^{* y}(j) & =\varphi^{*}(y+j)=\varphi^{*}(y)+\varphi^{* \pi^{*}(y)}(j)=\rho^{* \varphi^{*}(y)} \varphi^{* \pi^{*}(y)}(j) .
\end{aligned}
$$


These equations imply that the first equations in (3.5) and (3.6) hold if we set $\alpha(x)=\varphi(x)$, $\beta(x)=\pi(x), \alpha^{*}(y)=\varphi^{*}(y)$ and $\beta^{*}(y)=\pi^{*}(y)$.

Employing induction, from the first equations in (3.5) and (3.6) we derive that

$$
\varphi^{k} \rho^{u}=\rho^{\alpha^{k}(u)} \varphi^{\tau(u, k)} \quad \text { and } \quad \varphi^{* l} \rho^{* v}=\rho^{* \alpha^{* l}(v)} \tau^{* \tau^{*}(v, l)},
$$

where

$$
\tau(u, k)=\sum_{i=1}^{k} \beta\left(\alpha^{i-1}(u)\right) \quad \text { and } \quad \tau^{*}(v, l)=\sum_{i=1}^{l} \beta^{*}\left(\alpha^{* i-1}(v)\right) .
$$

By inverting the identities we obtain

$$
\rho^{-u} \varphi^{-k}=\varphi^{-\tau(u, k)} \rho^{-\alpha^{k}(u)} \quad \text { and } \quad \rho^{*-v} \varphi^{*-l}=\varphi^{*-\tau^{*}(v, l)} \rho^{*-\alpha^{* l}(v)} .
$$

In particular,

$$
\rho \varphi^{y}=\varphi^{-\tau(-1,-y)} \rho^{-\alpha^{-y}(-1)} \quad \text { and } \quad \rho^{*} \varphi^{* x}=\varphi^{*-\tau^{*}(-1,-x)} \rho^{*-\alpha^{*-x}(-1)} .
$$

Recall that

$$
\beta(x)=\pi(x)=-\varphi^{*-x}(-1)=-\alpha^{*-x}(-1)
$$

and

$$
\beta^{*}(y)=\pi^{*}(y)=-\varphi^{-y}(-1)=-\alpha^{-y}(-1) .
$$

Thus the second equations in (3.5) and (3.6) will hold if

$$
\alpha(x)=\varphi(x) \equiv-\tau^{*}(-1,-x) \quad\left(\bmod \left|\varphi^{*}\right|\right)
$$

and

$$
\alpha^{*}(y)=\varphi^{*}(y) \equiv-\tau(-1,-y) \quad(\bmod |\varphi|) .
$$

Indeed, by Lemma 3.1(iii) we have $\tau^{*}\left(-1,\left|\varphi^{*}\right|\right) \equiv 0\left(\bmod \left|\varphi^{*}\right|\right)$. Since

$$
\begin{aligned}
\tau^{*}\left(-1,\left|\varphi^{*}\right|\right) & =\sum_{i=1}^{\left|\varphi^{*}\right|} \beta^{*}\left(\alpha^{* i-1}(-1)\right)=\sum_{i=1}^{\left|\varphi^{*}\right|} \pi^{*}\left(\varphi^{* i-1}(-1)\right) \\
& =\sum_{i=1}^{\left|\varphi^{*}\right|-x} \pi^{*}\left(\varphi^{* i-1}(-1)\right)+\sum_{i=\left|\varphi^{*}\right|-x+1}^{\left|\varphi^{*}\right|} \pi^{*}\left(\varphi^{* i-1}(-1)\right) \\
& =\tau^{*}(-1,-x)+\sum_{i=1}^{x} \pi^{*}\left(\varphi^{*-i}(-1)\right) \quad\left(\bmod \left|\varphi^{*}\right|\right)
\end{aligned}
$$

we obtain

$$
-\sigma^{*}(-1,-x) \equiv \sum_{i=1}^{x} \pi^{*}\left(\varphi^{*-i}(-1)\right) \quad\left(\bmod \left|\varphi^{*}\right|\right) .
$$

On the other hand, since $\varphi$ is a skew-morphism of $\mathbb{Z}_{m}$, we have $\varphi(z-1)=\varphi(z)+$ $\varphi^{\pi(z)}(-1)$ for all $z \in \mathbb{Z}_{n}$, so $\varphi(z-1)-\varphi(z)=\varphi^{\pi(z)}(-1)$. By combining these identities 
we obtain

$$
\begin{aligned}
\varphi(x) & =-(\varphi(0)-\varphi(x))=-\sum_{i=1}^{x}(\varphi(i-1)-\varphi(i))=-\sum_{i=1}^{x} \varphi^{\pi(i)}(-1) \\
& =-\sum_{i=1}^{x} \varphi^{-\varphi^{*-i}(-1)}(-1)=\sum_{i=1}^{x} \pi^{*}\left(\varphi^{*-i}(-1)\right) \equiv-\sigma^{*}(-1,-x) \quad\left(\bmod \left|\varphi^{*}\right|\right) .
\end{aligned}
$$

Thus we have shown that

$$
\varphi(x) \equiv-\sigma^{*}(-1,-x) \equiv \sum_{i=1}^{x} \pi^{*}\left(\varphi^{*-i}(-1)\right) \quad\left(\bmod \left|\varphi^{*}\right|\right) .
$$

By using similar arguments we can prove that $\alpha^{*}(y)=\varphi^{*}(y) \equiv-\sigma(-1,-y)(\bmod |\varphi|)$. Thus, $\langle a\rangle\langle b\rangle$ is a subgroup of $G$, as claimed.

Finally, since $G=\langle a, b\rangle$, we have $G=\langle a\rangle\langle b\rangle$, so $(G ; a, b)$ is an exact $(m, n)$-bicyclic triple. Note that $a b^{x}=b^{\alpha(x)} a^{\beta(x)}$ and $b a^{y}=a^{\alpha^{*}(y)} b^{\beta^{*}(y)}$ with $\alpha(x)=\varphi(x)$ and $\alpha^{*}(y)=$ $\varphi^{*}(y)$. It follows that $\varphi$ and $\varphi^{*}$ are precisely the skew-morphisms induced by $a$ and $b$ in the triple $(G ; a, b)$.

Putting together Theorem 2.1, Proposition 3.3, and Proposition 3.4 we obtain a oneto-one correspondence between $(m, n)$-complete regular dessins, exact $(m, n)$-bicyclic triples, and $(m, n)$-reciprocal pairs of skew-morphisms.

Theorem 3.5. For every pair of positive integers $m$ and $n$ there exists a one-to-one correspondence between any two sets of the following three types of objects:

(i) isomorphism classes of $(m, n)$-complete regular dessins,

(ii) equivalence classes of exact $(m, n)$-bicyclic triples, and

(iii) $(m, n)$-reciprocal pairs of skew-morphisms.

Proof. The correspondence between the isomorphism classes of $(m, n)$-complete regular dessins and equivalence classes of exact $(m, n)$-bicyclic triples has been established in Theorem 2.1. It remains to prove that there is a one-to-one correspondence between equivalence classes of exact $(m, n)$-bicyclic triples and $(m, n)$-reciprocal pairs of skewmorphisms.

By Proposition 3.3, every exact $(m, n)$-bicyclic triple $(G ; a, b)$ determines an $(m, n)$ reciprocal pair $\left(\varphi, \varphi^{*}\right)$ of skew-morphisms of $\mathbb{Z}_{n}$ and $\mathbb{Z}_{m}$. Conversely, by Proposition 3.4, every $(m, n)$-reciprocal pair $\left(\varphi, \varphi^{*}\right)$ of skew-morphisms determines an exact $(m, n)$-bicyclic triple $(G ; a, b)$, and the pair of skew-morphisms induced by the elements $a$ and $b$ in this triple is identical to the original one. What remains to prove is the one-to-one correspondence.

If two $(m, n)$-reciprocal pairs $\left(\varphi_{1}, \varphi_{1}^{*}\right)$ and $\left(\varphi_{2}, \varphi_{2}^{*}\right)$ are identical, then clearly so will be the corresponding $(m, n)$-bicyclic triples. Conversely, let $\left(G_{1} ; a_{1}, b_{1}\right)$ and $\left(G_{2} ; a_{2}, b_{2}\right)$ be two equivalent exact $(m, n)$-bicyclic triples, and let $\left(\varphi_{1}, \varphi_{1}^{*}\right)$ and $\left(\varphi_{2}, \varphi_{2}^{*}\right)$ be the corresponding skew-morphisms. Since $\left(G_{1} ; a_{1}, b_{1}\right)$ and $\left(G_{2} ; a_{2}, b_{2}\right)$ are equivalent, the assignment $\theta: a_{1} \mapsto a_{2}, b_{1} \mapsto b_{2}$ extends to an isomorphism of $G_{1}$ to $G_{2}$; in particular, $\left|a_{1}\right|=\left|a_{2}\right|$ and $\left|b_{1}\right|=\left|b_{2}\right|$. Set $m=\left|a_{1}\right|$ and $n=\left|b_{1}\right|$. Recall that the skew-morphisms $\varphi_{1}$ and $\varphi_{2}$ induced by $a_{1}$ and $a_{2}$ are determined by the rules $a_{1} b_{1}^{x}=b_{1}^{\varphi_{1}(x)} a_{1}^{\pi_{1}(x)}$ and 
$a_{2} b_{2}^{y}=b_{2}^{\varphi_{2}(y)} a_{2}^{\pi_{2}(y)}$ where $x, y \in \mathbb{Z}_{n}$. If we apply the isomorphism $\theta$ to the first equation we obtain $a_{2} b_{2}^{x}=\theta\left(a_{1} b_{1}^{x}\right)=\theta\left(b_{1}^{\varphi_{1}(x)} a_{1}^{\pi_{1}(x)}\right)=b_{2}^{\varphi_{1}(x)} a_{2}^{\pi_{1}(x)}$, and combining this with the second equation we get $b_{2}^{\varphi_{2}(x)} a_{2}^{\pi_{2}(x)}=b_{2}^{\varphi_{1}(x)} a_{2}^{\pi_{1}(x)}$. Thus $\varphi_{1}=\varphi_{2}$. Using similar arguments we can get $\varphi_{1}^{*}=\varphi_{2}^{*}$. Hence, $\left(\varphi_{1}, \varphi_{1}^{*}\right)=\left(\varphi_{2}, \varphi_{2}^{*}\right)$.

In the course of the proof of Proposition 3.4 we have established the identity (3.7). The following corollary makes it explicit.

Corollary 3.6. If $\left(\varphi, \varphi^{*}\right)$ is an $(m, n)$-reciprocal pair of skew-morphisms, then $\varphi$ and $\varphi^{*}$ satisfy the following identities:

$\varphi(x)=\sum_{i=1}^{x} \pi^{*}\left(\varphi^{*-i}(-1)\right) \quad\left(\bmod \left|\varphi^{*}\right|\right) \quad$ and $\quad \varphi^{*}(y)=\sum_{i=1}^{y} \pi\left(\varphi^{-i}(-1)\right) \quad(\bmod |\varphi|)$.

Next we offer two examples. The first of them deals with the standard $(m, n)$-complete dessins.

Example 3.7. Let us revisit the group $G=\left\langle a, b \mid a^{m}=b^{n}=[a, b]=1\right\rangle \cong \mathbb{Z}_{m} \times \mathbb{Z}_{n}$ considered in Example 2.2 and determine all reciprocal pairs of skew-morphisms arising from $G$. Obviously, $G$ gives rise to only one equivalence class of bicyclic triples, so we only need to consider the pairs of skew-morphisms induced by $a$ and $b$ in the triple $(G ; a, b)$. By checking the identities (3.2), we immediately see that the skew-morphisms are the identity automorphisms. Thus the only reciprocal pair of skew-morphisms arising from the group $\mathbb{Z}_{m} \times \mathbb{Z}_{n}$ is $\left(\mathrm{id}_{n}, \mathrm{id}_{m}\right)$, where $\mathrm{id}_{n}: \mathbb{Z}_{n} \rightarrow \mathbb{Z}_{n}$ and id $\mathrm{id}_{m}: \mathbb{Z}_{m} \rightarrow \mathbb{Z}_{m}$ denote the identity mappings. In other words, for every pair of positive integers $m$ and $n$ there exists only one complete dessin whose automorphism group is isomorphic to the direct product $\mathbb{Z}_{m} \times \mathbb{Z}_{n}$, the standard $(m, n)$-complete dessin.

In the next example, which is extracted from [14], we present a complete list of pairs of reciprocal skew-morphisms of the cyclic groups $\mathbb{Z}_{9}$ and $\mathbb{Z}_{27}$.

Example 3.8. In order to list all reciprocal pairs $\left(\varphi, \varphi^{*}\right)$ of skew-morphisms $\varphi: \mathbb{Z}_{9} \rightarrow \mathbb{Z}_{9}$ and $\varphi^{*}: \mathbb{Z}_{27} \rightarrow \mathbb{Z}_{27}$ let us first observe that $\varphi$ must be an automorphism. Indeed, the order of $\varphi$ divides 27 , so $|\varphi|=1$ or $|\varphi|=3$. If $|\varphi|=1$, then $\varphi$ is an identity automorphism. If $\varphi$ has order 3 and is not an automorphism, then the power function of $\varphi$ reduced to $\mathbb{Z}_{3}$ can take only two values 1 and 2 , so the subgroup $\operatorname{ker} \varphi$ must have index 2 in $\mathbb{Z}_{3}$, which is impossible. This proves that $\varphi$ is an automorphism.

Now, there are exactly 27 reciprocal pairs of skew-morphisms $\left(\varphi, \varphi^{*}\right)$ of skew-morphisms $\varphi: \mathbb{Z}_{9} \rightarrow \mathbb{Z}_{9}$ and $\varphi^{*}: \mathbb{Z}_{27} \rightarrow \mathbb{Z}_{27}$, falling into one of the following two types:

(i) Both $\varphi$ and $\varphi^{*}$ are group automorphisms: In this case $\varphi(x) \equiv e x(\bmod 9)$ and $\varphi^{*}(y) \equiv f y(\bmod 27)$ where either $e=1$ and $f \in\{1,4,7,10,13,16,19,22,25\}$, or $e \in\{4,7\}$ and $f \in\{1,10,19\}$. Thus there are $9+6=15$ reciprocal pairs of skew-morphisms of this type.

(ii) $\varphi$ is a group automorphism but $\varphi^{*}$ is not: In this case $\varphi(x) \equiv \operatorname{ex}(\bmod 9)$ and $\varphi^{*}(y) \equiv y+3 t \sum_{i=1}^{y} \sigma\left(s, e^{i-1}\right)(\bmod 27)$ where $e \in\{4,7\}$ and $\sigma\left(s, e^{i-1}\right)=$ $\sum_{j=1}^{e^{i-1}} s^{j-1}$ where $(s, t)=(4,1),(7,2),(4,4),(7,5),(4,7)$ or $(7,8)$. There are $2 \times$ $6=12$ reciprocal pairs of this type. 
We remark that in [14, Theorem 14] all reciprocal pairs of skew-morphisms of cyclic groups are classified provided that one of the skew-morphisms is an automorphism.

The correspondence established in Theorem 3.5 implies that the second condition required in the definition of an $(m, n)$-reciprocal pair of skew-morphisms (see Definition 3.2) can be replaced with a simpler condition.

Corollary 3.9. A pair $\left(\varphi, \varphi^{*}\right)$ of skew-morphisms $\varphi: \mathbb{Z}_{n} \rightarrow \mathbb{Z}_{n}$ and $\varphi^{*}: \mathbb{Z}_{m} \rightarrow \mathbb{Z}_{m}$ with power functions $\pi$ and $\pi^{*}$, respectively, is reciprocal if and only if the following two conditions are satisfied:

(i) $|\varphi|$ divides $m$ and $\left|\varphi^{*}\right|$ divides $n$, and

(ii) $\pi(x)=\varphi^{* x}(1)$ and $\pi^{*}(y)=\varphi^{y}(1)$.

Proof. It is sufficient to replace the original dessin, represented by an exact $(m, n)$-bicyclic triple $(G ; a, b)$, with its mirror image, for which the corresponding bicyclic triple is $\left(G ; a^{-1}, b^{-1}\right)$, and use Theorem 3.5.

\section{The uniqueness theorem}

We have seen in Example 3.7 that for each pair of positive integers $m$ and $n$ there exists, up to reciprocality and isomorphism, at least one complete regular dessin with the underlying graph $K_{m, n}$, namely, the standard $(m, n)$-complete dessin. In this section we determine all the pairs $(m, n)$ for which the standard $(m, n)$-complete dessin is the only regular $(m, n)$ dessin.

A pair $(m, n)$ of positive integers $m$ and $n$ will be called singular if

$$
\operatorname{gcd}(m, \phi(n))=\operatorname{gcd}(n, \phi(m))=1 \text {. }
$$

A positive integer $n$ will be called singular if the pair $(n, n)$ is singular, that is, if $\operatorname{gcd}(n, \phi(n))=1$. We now show that for each non-singular pair $(m, n)$ of positive integers there exists a non-abelian exact $(m, n)$-bicyclic group.

Example 4.1. Let $m$ and $n$ be positive integers. First assume that $\operatorname{gcd}(n, \phi(m)) \neq 1$. It is well known that for $x \in \mathbb{Z}_{m}$ the assignment $1 \mapsto x$ extends to an automorphism of $\mathbb{Z}_{m}$ if and only if $\operatorname{gcd}(x, m)=1$, and thus $\left|\operatorname{Aut}\left(\mathbb{Z}_{m}\right)\right|=\phi(m)$. Since $\operatorname{gcd}(n, \phi(m)) \neq 1$, there exists an integer $r$ such that $r \not \equiv 1(\bmod m)$ and $r^{p} \equiv 1(\bmod m)$, where $p$ $\operatorname{gcd}(n, \phi(m))$. Define a group $G$ with presentation

$$
G=\left\langle a, b \mid a^{m}=b^{n}=1, b^{-1} a b=a^{r}\right\rangle .
$$

By Hölder's theorem [22, Chapter 7], $G$ is a well-defined metacyclic group of order $m n$. Since $r \not \equiv 1(\bmod m)$, the group $G$ is non-abelian. If $\operatorname{gcd}(m, \phi(n)) \neq 1$, we proceed similarly. Thus, whenever $(m, n)$ is non-singular, there always exists at least one nonabelian exact $(m, n)$-bicyclic group.

We remark that the argument used here is different from the one employed in the proof of Lemma 3.1 in [12].

We now apply our theory to proving the following theorem, which extends the validity of a result of Fan and Li [12] to all bicyclic groups, not just exact ones. 
Theorem 4.2. The following statements are equivalent for every pair of positive integers $m$ and $n$ :

(i) Every product of a cyclic group of order $m$ with a cyclic group of order $n$ is abelian.

(ii) The pair $(m, n)$ is singular.

Proof. If (i) holds, then by virtue of Example 4.1 the pair $(m, n)$ must be singular. For the converse, assume that the pair $(m, n)$ is singular and that $G$ is an $(m, n)$-bicyclic group. We prove the statement by using induction on the size of $|G|$. By a result of Huppert [15] and Douglas [8] (see also [17, VI.10.1]), $G$ is supersolvable, so for the largest prime factor $p$ of $|G|$ the Sylow $p$-subgroup $P$ of $G$ is normal in (see [17, VI.9.1]). By the Schur-Zassenhaus theorem, $G$ is a semidirect product of $P$ by $Q$, where $Q$ is a subgroup of order $|G / P|$ in $G$. To proceed we distinguish two cases.

Case 1. $p$ divides only one of $m$ and $n$. Without loss of generality we may assume that $p \mid m$ and $p \nmid n$. Let us write $m$ in the form $m=p^{e} m_{1}$ where $p \nmid m_{1}$. Then the normal subgroup $P$ is contained in the cyclic factor $A=\langle a\rangle$ of $G$ of order $m$, so $P=\left\langle a^{m_{1}}\right\rangle$. The generator $b$ of the cyclic factor $B=\langle b\rangle$ of order $n$ induces an automorphism $a^{m_{1}} \mapsto\left(a^{m_{1}}\right)^{r}$ of $P$ by conjugation $b^{-1} a^{m_{1}} b=\left(a^{m_{1}}\right)^{r}$ where $r$ is an integer coprime to $p$. It follows that the multiplicative order $|r|$ of $r$ in $\mathbb{Z}_{p^{e}}$ divides $|\operatorname{Aut}(P)|=\phi\left(p^{e}\right)$. On the other hand, $a^{m_{1}}=b^{-n} a^{m_{1}} b^{n}=\left(a^{m_{1}}\right)^{r^{n}}$, so $r^{n} \equiv 1\left(\bmod p^{e}\right)$, and hence $|r|$ also divides $n$. But $\phi\left(p^{e}\right)$ divides $\phi(m)$ and $\operatorname{gcd}(n, \phi(m))=1$, so $r \equiv 1\left(\bmod p^{e}\right)$. Therefore $P$ is contained in the centre of $G$, and hence $G=P \times Q$, where $Q$ is an $\left(m_{1}, n\right)$-bicyclic group. It is evident that the pair $\left(m_{1}, n\right)$ is also singular. By induction, $Q$ is abelian, and therefore $G$ is abelian.

Case 2. $p$ divides both $m$ and $n$. Since $(m, n)$ is a singular pair, $p^{2} \nmid m$ and $p^{2} \nmid n$. Thus $m=p m_{1}$ and $n=p n_{1}$ where $p \nmid m_{1}, p \nmid n_{1}$ and $\operatorname{gcd}\left(m_{1}, p(p-1)\right)=\operatorname{gcd}\left(n_{1}, p(p-1)\right)=$ 1. Since $|G|=|A B|=|A||B| /|A \cap B|$, the Sylow $p$-subgroup $P$ of $G$ is of order $p$ or $p^{2}$. If $p$ divides $|A \cap B|$, then $|P|=p$ and so $P \leq A \cap B$, which is central in $G$. Therefore, $G=P \times Q$, where $Q$ is an $\left(m_{1}, n_{1}\right)$-bicyclic group, and the result follows by induction. Otherwise, $p \nmid|A \cap B|$, so $P \cong \mathbb{Z}_{p} \times \mathbb{Z}_{p}$. We may view $P$ as a 2-dimensional vector space over the Galois field $\mathbb{F}_{p}$. Let $\Omega$ be the set of 1-dimensional subspaces of $P$. Then $|\Omega|=p+1$ and $\alpha=\left\langle a^{m_{1}}\right\rangle$ belongs to $\Omega$. Consider the action of $G$ on $P$ by conjugation. The kernel of this action is $C_{G}(P)$, so $\bar{G}=G / C_{G}(P) \leq \mathrm{GL}(2, p)$ where $C_{G}(P)$ denotes the centraliser of $P$ in $G$. Now we claim that $\bar{G}=1$.

Suppose to the contrary that $\bar{G} \neq 1$. Since $G=\langle a, b\rangle$, we have $\bar{G}=\left\langle\bar{a}^{p}, \bar{b}^{p}\right\rangle$, where $\bar{a}^{p}=a^{p} C_{G}(P)$ and $\bar{b}^{p}=b^{p} C_{G}(P)$. Hence at least one of $\bar{a}^{p}$ and $\bar{b}^{p}$ is not the identity of $\bar{G}$, say $\bar{a}^{p} \neq 1$. Clearly, $\left|\bar{a}^{p}\right|$ divides $m_{1}$, the order of $a^{p}$ in $G$.

Note that $\Omega$ is a complete block system of $\operatorname{GL}(2, p)$ on $P$ and the induced action of $\mathrm{GL}(2, p)$ on $\Omega$ is transitive. By the Frattini argument, $|\mathrm{GL}(2, p)|=(p+1)\left|\mathrm{GL}(2, p)_{\alpha}\right|$, and hence $\left|\mathrm{GL}(2, p)_{\alpha}\right|=p(p-1)^{2}$ as $|\mathrm{GL}(2, p)|=p(p+1)(p-1)^{2}$. On the other hand, $\bar{a}^{p}$ fixes $\alpha$ as $a$ fixes the subspace $\langle a\rangle$, implying that $\bar{a}^{p} \in \mathrm{GL}(2, p)_{\alpha}$. It follows that $\left|\overline{a^{p}}\right|$ divides $p(p-1)^{2}$. Since $\left|\overline{a^{p}}\right|$ divides $m_{1}$ and $\operatorname{gcd}\left(m_{1}, p(p-1)\right)=1$, we have $\left|\overline{a^{p}}\right|=1$, which is impossible because $\bar{a}^{p} \neq 1$. Thus $\bar{G}=1$, as claimed.

Since $\bar{G}=1$, we have $G=C_{G}(P)$, and hence $G=P \times Q$, where $Q=\left\langle a^{p}\right\rangle\left\langle b^{p}\right\rangle$ is an $\left(m_{1}, n_{1}\right)$-bicyclic group with the pair $\left(m_{1}, n_{1}\right)$ being singular. The statement now follows by induction.

The following result follows easily from Theorem 4.2. 
Corollary 4.3. Let $m$ and $n$ be positive integers. Then every group factorisable as an exact product of cyclic subgroups of orders $m$ and $n$ is abelian if and only if the pair $(m, n)$ is singular.

We summarize the results of this section in the following theorem.

Theorem 4.4. The following statements are equivalent for any pair of positive integers $m$ and $n$ :

(i) The pair $(m, n)$ is singular.

(ii) Every finite group factorisable as a product of two cyclic subgroups of orders $m$ and $n$ is abelian.

(iii) Every finite group factorisable as an exact product of two cyclic groups of orders $m$ and $n$ is isomorphic to $\mathbb{Z}_{m} \times \mathbb{Z}_{n}$.

(iv) There is only one $(m, n)$-reciprocal pair of skew-morphisms $\left(\varphi, \varphi^{*}\right)=\left(\mathrm{id}_{n}, \mathrm{id}_{m}\right)$ of the cyclic groups $\mathbb{Z}_{n}$ and $\mathbb{Z}_{m}$.

(v) Up to reciprocality, there is a unique isomorphism class of regular dessins whose underlying graph is the complete bipartite graph $K_{m, n}$.

(vi) There exists a unique isomorphism class of orientable edge-transitive embeddings of $K_{m, n}$.

The proof of the equivalence between items (i), (iii) and (vi) of Theorem 4.4 can be found in [12, Theorem 1.1].

Remark 4.5. For a fixed positive integer $x$, it has been recently shown by Nedela and Pomerance [39] that the number of singular pairs $(m, n)$ with $m, n \leq x$ is asymptotic to $z(x)^{2}$ where

$$
z(x)=e^{\gamma} \frac{x}{\log \log \log x},
$$

where $\gamma$ is Euler's constant.

\section{The symmetric case}

Recall that a complete regular dessin $\mathcal{D}=(G ; a, b)$ is symmetric if $G$ has an automorphism transposing $a$ and $b$. In this case the dessin $\mathcal{D}$ possesses an external symmetry transposing the colour-classes. If we ignore the vertex-colouring, the dessin can be regarded as an orientably regular map with underlying graph $K_{n, n}$. As a consequence of Theorem 3.5 we obtain the following correspondence between orientably regular embeddings of the complete bipartite graphs $K_{n, n}$ and symmetric skew-morphisms of $\mathbb{Z}_{n}$, partially indicated by Kwak and Kwon already in [34, Lemma 3.5].

Corollary 5.1. The isomorphism classes of orientably regular embeddings of complete bipartite graphs $K_{n, n}$ are in a one-to-one correspondence with the symmetric skew-morphisms of $\mathbb{Z}_{n}$.

A complete classification of orientably regular embeddings of complete bipartite graphs $K_{n, n}$ has already been accomplished by Jones et al. in a series of papers [9, 10, 11, 23, 25, 26, 40]. The methods used in the classification rely on the analysis of the structure of the associated exact bicyclic groups. A different approach to the classification can be taken on 
the basis of Corollary 5.1 via determining the corresponding symmetric skew-morphisms of $\mathbb{Z}_{n}$. In particular, we can reformulate Theorem A of [23] as follows:

Corollary 5.2. The following statements are equivalent for every positive integer $n$ :

(i) The integer $n$ is singular.

(ii) Every finite group factorisable as a product of two cyclic subgroups of order $n$ is abelian.

(iii) Every finite group factorisable as an exact product of two cyclic subgroups of order $n$ is isomorphic to $\mathbb{Z}_{n} \times \mathbb{Z}_{n}$.

(iv) The cyclic group $\mathbb{Z}_{n}$ has only one symmetric skew-morphism.

(v) Up to isomorphism, the complete bipartite graph $K_{n, n}$ has a unique orientably regular embedding.

Although skew-morphisms are implicitly present in the structure of the automorphism groups of the maps, how to find them explicitly is not at all clear. This leads us to formulating the following problems for future investigation.

Problem 5.3. Determine the symmetric skew-morphisms of cyclic groups by means of explicit formulae.

Problem 5.4. Classify all orientably regular embeddings of complete bipartite graphs $K_{n, n}$ in terms of the corresponding symmetric skew-morphisms.

The previous problem suggests the following natural question: under what conditions a symmetric skew-morphism is a group automorphism and what are the corresponding orientably regular maps? The following result determines these skew-morphisms explicitly.

Theorem 5.5. Let $\varphi: x \mapsto r x$ be an automorphism of $\mathbb{Z}_{n}$ of order $d$, where $\operatorname{gcd}(r, n)=1$. Then $\varphi$ is a symmetric skew-morphism of $\mathbb{Z}_{n}$ if and only if $d \mid n$ and $r \equiv 1(\bmod d)$.

Proof. Note that the order of $\varphi$ is equal to the multiplicative order of $r$ in $\mathbb{Z}_{n}$. Since $\left|\operatorname{Aut}\left(\mathbb{Z}_{n}\right)\right|=\phi(n)$, we have $d \mid \phi(n)$. Since $\varphi$ is an automorphism, the associated power function is $\pi(x) \equiv 1(\bmod d)$ for all $x \in \mathbb{Z}_{n}$.

If $\varphi$ is symmetric, then by Definition $3.2, d \mid n$ and $\pi(x)=-\varphi^{-x}(-1)(\bmod d)$ for all $x \in \mathbb{Z}_{n}$. In particular, $1=\pi(-1) \equiv-\varphi(-1) \equiv \varphi(1) \equiv r(\bmod d)$.

Conversely, assume that $d \mid n$ and $r \equiv 1(\bmod d)$. By Definition 3.2, it suffices to show that $-\varphi^{-x}(-1)$ is a power function of $\varphi$ where $x \in \mathbb{Z}_{n}$, that is, to show that $-\varphi^{-x}(-1) \equiv 1(\bmod d)$. Since $r \equiv 1(\bmod d)$, we have $-\varphi^{-x}(-1)=\varphi^{-x}(1)=$ $r^{-x} \equiv 1(\bmod d)$, as required.

The following example shows that there exist symmetric skew-morphisms of $\mathbb{Z}_{n}$ which are not automorphisms.

Example 5.6. The cyclic group $\mathbb{Z}_{8}$ has the total of six skew-morphisms, out of which four are automorphisms and two are proper skew-morphisms. The latter two are listed below along with the corresponding power functions:

$$
\begin{aligned}
& \varphi=(0)(2)(4)(6)(1357) \text {, } \\
& \pi_{\varphi}=[1][1][1][1]\left[\begin{array}{llll}
3 & 3 & 3 & 3
\end{array}\right] ; \\
& \psi=(0)(2)(4)(6)(1753) \text {, } \\
& \pi_{\psi}=[1][1][1][1]\left[\begin{array}{llll}
3 & 3 & 3 & 3
\end{array}\right] \text {. }
\end{aligned}
$$


Note that they are, in fact, antiautomorphisms in the sense of [43, 44]. It can be easily verified that all the six skew-morphisms are symmetric. It follows that they correspond to the six non-isomorphic orientably regular embeddings of $K_{8,8}$ described in [25, Table 1].

\section{ORCID iDs}

Yan-Quan Feng (D) https://orcid.org/0000-0003-3214-0609

Kan Hu (D) https://orcid.org/0000-0003-4775-7273

Roman Nedela (D) https://orcid.org/0000-0002-9826-704X

Martin Škoviera (D) https://orcid.org/0000-0002-2108-7518

Na-Er Wang (D) https://orcid.org/0000-0002-0832-0717

\section{References}

[1] M. Bachratý and R. Jajcay, Powers of skew-morphisms, in: J. Širáň and R. Jajcay (eds.), Symmetries in Graphs, Maps, and Polytopes, Springer, Cham, volume 159 of Springer Proceedings in Mathematics \& Statistics, 2016 pp. 1-25, doi:10.1007/978-3-319-30451-9_1, papers from the 5th SIGMAP Workshop held in West Malvern, July 7 - 11, 2014.

[2] M. Bachratý and R. Jajcay, Classification of coset-preserving skew-morphisms of finite cyclic groups, Australas. J. Combin. 67 (2017), 259-280, https: / / a jc.maths . uq. edu . au/ pdf/67/ajc_v67_p259.pdf.

[3] G. V. Belyı̆, Galois extensions of a maximal cyclotomic field, Izv. Akad. Nauk SSSR Ser. Mat. 43 (1979), 267-276, http: / /mi . mathnet. ru/izv1682.

[4] M. Conder, R. Jajcay and T. Tucker, Regular Cayley maps for finite abelian groups, J. Algebraic Combin. 25 (2007), 259-283, doi:10.1007/s10801-006-0037-0.

[5] M. D. E. Conder, R. Jajcay and T. W. Tucker, Cyclic complements and skew morphisms of groups, J. Algebra 453 (2016), 68-100, doi:10.1016/j.jalgebra.2015.12.024.

[6] M. D. E. Conder and T. W. Tucker, Regular Cayley maps for cyclic groups, Trans. Amer. Math. Soc. 366 (2014), 3585-3609, doi:10.1090/s0002-9947-2014-05933-3.

[7] A. D. Coste, G. A. Jones, M. Streit and J. Wolfart, Generalised Fermat hypermaps and Galois orbits, Glasg. Math. J. 51 (2009), 289-299, doi:10.1017/s0017089509004972.

[8] J. Douglas, On the supersolvability of bicyclic groups, Proc. Nat. Acad. Sci. U.S.A. 47 (1961), 1493-1495, doi:10.1073/pnas.47.9.1493.

[9] S. Du, G. Jones, J. H. Kwak, R. Nedela and M. Škoviera, 2-groups that factorise as products of cyclic groups, and regular embeddings of complete bipartite graphs, Ars Math. Contemp. 6 (2013), 155-170, doi:10.26493/1855-3974.295.270.

[10] S.-F. Du, G. Jones, J. H. Kwak, R. Nedela and M. Škoviera, Regular embeddings of $K_{n, n}$ where $n$ is a power of 2. I: Metacyclic case, European J. Combin. 28 (2007), 1595-1609, doi:10.1016/j.ejc.2006.08.012.

[11] S.-F. Du, G. Jones, J. H. Kwak, R. Nedela and M. Škoviera, Regular embeddings of $K_{n, n}$ where $n$ is a power of 2. II: The non-metacyclic case, European J. Combin. 31 (2010), 1946-1956, doi:10.1016/j.ejc.2010.01.009.

[12] W. Fan and C. H. Li, The complete bipartite graphs with a unique edge-transitive embedding, J. Graph Theory 87 (2018), 581-586, doi:10.1002/jgt.22176.

[13] G. González-Diez and A. Jaikin-Zapirain, The absolute Galois group acts faithfully on regular dessins and on Beauville surfaces, Proc. Lond. Math. Soc. 111 (2015), 775-796, doi:10.1112/ plms/pdv041. 
[14] K. Hu, R. Nedela, N.-E. Wang and K. Yuan, Reciprocal skew morphisms of cyclic groups, Acta Math. Univ. Comenian. (N.S.) 88 (2019), 305-318, http: / / www . iam. fmph . uniba.sk/ amuc/ojs/index.php/amuc/article/view/1006.

[15] B. Huppert, Über das Produkt von paarweise vertauschbaren zyklischen Gruppen, Math. Z. 58 (1953), 243-264, doi:10.1007/bf01174144.

[16] B. Huppert, Über die Auflösbarkeit faktorisierbarer Gruppen, Math. Z. 59 (1953), 1-7, doi: $10.1007 / \mathrm{bf01180236.}$

[17] B. Huppert, Endliche Gruppen I, volume 134 of Die Grundlehren der Mathematischen Wissenschaften, Springer-Verlag, Berlin-New York, 1967, doi:10.1007/978-3-642-64981-3.

[18] N. Itô, Über das Produkt von zwei abelschen Gruppen, Math. Z. 62 (1955), 400-401, doi: $10.1007 / \mathrm{bf01180647.}$

[19] R. Jajcay and R. Nedela, Half-regular Cayley maps, Graphs Combin. 31 (2015), 1003-1018, doi:10.1007/s00373-014-1428-y.

[20] R. Jajcay and J. Širáň, Skew-morphisms of regular Cayley maps, Discrete Math. 244 (2002), 167-179, doi:10.1016/s0012-365x(01)00081-4.

[21] Z. Janko, Finite 2-groups with exactly one nonmetacyclic maximal subgroup, Israel J. Math. 166 (2008), 313-347, doi:10.1007/s11856-008-1033-y.

[22] D. L. Johnson, Presentations of Groups, volume 15 of London Mathematical Society Student Texts, Cambridge University Press, Cambridge, 2nd edition, 1997, doi:10.1017/ cbo9781139168410.

[23] G. Jones, R. Nedela and M. Škoviera, Complete bipartite graphs with a unique regular embedding, J. Comb. Theory Ser. B 98 (2008), 241-248, doi:10.1016/j.jctb.2006.07.004.

[24] G. Jones and D. Singerman, Belyu functions, hypermaps and Galois groups, Bull. London Math. Soc. 28 (1996), 561-590, doi:10.1112/blms/28.6.561.

[25] G. A. Jones, Regular embeddings of complete bipartite graphs: classification and enumeration, Proc. Lond. Math. Soc. (3) 101 (2010), 427-453, doi:10.1112/plms/pdp061.

[26] G. A. Jones, R. Nedela and M. Škoviera, Regular embeddings of $K_{n, n}$ where $n$ is an odd prime power, European J. Combin. 28 (2007), 1863-1875, doi:10.1016/j.ejc.2005.07.021.

[27] G. A. Jones, M. Streit and J. Wolfart, Galois action on families of generalised Fermat curves, J. Algebra 307 (2007), 829-840, doi:10.1016/j.jalgebra.2006.10.009.

[28] G. A. Jones and J. Wolfart, Dessins d'Enfants on Riemann Surfaces, Springer Monographs in Mathematics, Springer, Cham, 2016, doi:10.1007/978-3-319-24711-3.

[29] I. Kovács and Y. S. Kwon, Regular Cayley maps on dihedral groups with the smallest kernel, J. Algebraic Combin. 44 (2016), 831-847, doi:10.1007/s10801-016-0689-3.

[30] I. Kovács and Y. S. Kwon, Classification of reflexible Cayley maps for dihedral groups, J. Comb. Theory Ser. B 127 (2017), 187-204, doi:10.1016/j.jctb.2017.06.002.

[31] I. Kovács, D. Marušič and M. Muzychuk, On $G$-arc-regular dihedrants and regular dihedral maps, J. Algebraic Combin. 38 (2013), 437-455, doi:10.1007/s10801-012-0410-0.

[32] I. Kovács and R. Nedela, Decomposition of skew-morphisms of cyclic groups, Ars Math. Contemp. 4 (2011), 329-349, doi:10.26493/1855-3974.157.fc1.

[33] I. Kovács and R. Nedela, Skew-morphisms of cyclic p-groups, J. Group Theory 20 (2017), 1135-1154, doi:10.1515/jgth-2017-0015.

[34] J. H. Kwak and Y. S. Kwon, Regular orientable embeddings of complete bipartite graphs, J. Graph Theory 50 (2005), 105-122, doi:10.1002/jgt.20097. 
[35] J. H. Kwak, Y. S. Kwon and R. Feng, A classification of regular $t$-balanced Cayley maps on dihedral groups, European J. Combin. 27 (2006), 382-393, doi:10.1016/j.ejc.2004.12.002.

[36] Y. S. Kwon, A classification of regular $t$-balanced Cayley maps for cyclic groups, Discrete Math. 313 (2013), 656-664, doi:10.1016/j.disc.2012.12.012.

[37] S. K. Lando and A. K. Zvonkin, Graphs on Surfaces and Their Applications, volume 141 of Encyclopaedia of Mathematical Sciences, Springer-Verlag, Berlin, 2004, doi:10.1007/ 978-3-540-38361-1.

[38] S. Lang, Introduction to Algebraic and Abelian Functions, Addison-Wesley, Reading, Massachusetts, 1972.

[39] R. Nedela and C. Pomerance, Density of singular pairs of integers, Integers 18 (2018), Paper No. A82 (7 pages), http://math. colgate.edu/ integers/s82/s82.mail. html.

[40] R. Nedela, M. Škoviera and A. Zlatoš, Regular embeddings of complete bipartite graphs, Discrete Math. 258 (2002), 379-381, doi:10.1016/s0012-365x(02)00539-3.

[41] D. J. S. Robinson, A Course in the Theory of Groups, volume 80 of Graduate Texts in Mathematics, Springer-Verlag, New York, 2nd edition, 1996, doi:10.1007/978-1-4419-8594-1.

[42] L. Schneps and P. Lochak (eds.), Geometric Galois Actions, Volume 1, volume 242 of London Mathematical Society Lecture Note Series, Cambridge University Press, Cambridge, 1997, doi: 10.1017/cbo9780511758874.

[43] J. Širáň and M. Škoviera, Groups with sign structure and their antiautomorphisms, Discrete Math. 108 (1992), 189-202, doi:10.1016/0012-365x(92)90674-5.

[44] J. Širáň and M. Škoviera, Regular maps from Cayley graphs. II. Antibalanced Cayley maps, Discrete Math. 124 (1994), 179-191, doi:10.1016/0012-365x(94)90089-2.

[45] N.-E. Wang, K. Hu, K. Yuan and J.-Y. Zhang, Smooth skew morphisms of dihedral groups, Ars Math. Contemp. 16 (2019), 527-547.

[46] J.-Y. Zhang, A classification of regular Cayley maps with trivial Cayley-core for dihedral groups, Discrete Math. 338 (2015), 1216-1225, doi:10.1016/j.disc.2015.01.036.

[47] J.-Y. Zhang, Regular Cayley maps of skew-type 3 for dihedral groups, Discrete Math. 338 (2015), 1163-1172, doi:10.1016/j.disc.2015.01.038.

[48] J.-Y. Zhang and S. Du, On the skew-morphisms of dihedral groups, J. Group Theory 19 (2016), 993-1016, doi:10.1515/jgth-2016-0027. 